Letter to the Editor

\title{
The Role of Glucose Transporter I in Drug Delivery: Transporter, Receptor and/or Signaling Component
}

\author{
Shiva Mehran ${ }^{1}$, Zafar Gholinejad $^{2}$ \\ ${ }^{1}$ Department of Biology, Higher Education Institute of Rabe-Rashidi, Tabriz, Iran. \\ ${ }^{2}$ Department of Medical Laboratory Science, Urmia Branch, Islamic Azad University, Urmia, Iran. \\ Corresponding author. E-mail: Zafar.gholinejad777@gmail.com, Ghzafar@yahoo.com Tel.: +98 4433676383 Fax: +98 4432780800
}

Received: Aug. 22, 2019; Accepted: Nov. 14, 2019; Published: Nov. 14, 2019.

Citation: Shiva Mehran, Zafar Gholinejad, The Role of Glucose Transporter 1 in Drug Delivery: Transporter, Receptor and/or Signaling Component. Nano Biomed. Eng., 2019, II (4): 347-350.

DOI: $10.5101 /$ nbe.v11i4.p347-350.

\begin{abstract}
The targeting of cell surface molecules is a promising strategy in drug delivery. Glucose modifiednanocarrier interacts with glucose transporter 1 (GLUT1) as a target molecule and increases nanocarrier-drug internalization to cancer and brain endothelial cells. The mechanism by which GLUT1 promotes nanocarrier internalization remains unclear. Herein, we propose that the interaction of GLUT1 and nanocarrier absorbs and traps the nanocarrier and simultaneously activates cell signaling pathways that is responsible for activation of the endocytosis system.
\end{abstract}

Keywords: GLUT1; Nanocarrier; Drug delivery; Signaling pathway

\section{Introduction}

Nonspecific-drug delivery is a challenging problem in nanoparticle-based drug delivery. The uptake of nanoparticles from blood is higher in cancer than normal tissue, because the endothelial of cancer vessels shows higher permeability and leakage [1]. This mechanism of drug delivery system is known as passive targeting [2]. Endothelial permeability and dysfunction are not observed in all cancers; therefore, passive targeting is not effective in all cancer types and leads to the development of another strategy known as active targeting. In active targeting, specific molecules are targeted in cancer tissues, which are differentially expressed between normal and malignant tissues. Moieties on the nanoparticle serve as a ligand that targets the receptors on the cancer tissue [3]. More than fifteen molecules have been targeted in order to deliver chemotherapeutic drugs in cancer therapy, including transferrin receptors, integrins, growth factor receptors, cluster of differentiation (CD) markers, glucose transporters (GLUTs), and asialoglycoprotein receptors [4]. Glucose and other monosaccharide are conjugated to nanocarrier for targeting GLUTs and asialoglycoprotein receptor. In nanoparticle-based drug delivery setting, these modification and targeting receptors increase nanoparticle internalization and delivery rate [5]. Fig. 1 summarizes the role of GLUT1 in cancer.

\section{Glucose Transporter 1}

Glucose transporter 1 (GLUT1) is overexpressed in several cancers. GLUT1 gene contains a Cis element recognized by a hypoxia-inducible factor 1 transcription factor that enhances GLUT1 expression in the cancer cells [6]. GLUT1 plays critical role in cancer progression by inducing of glucose uptake. The 


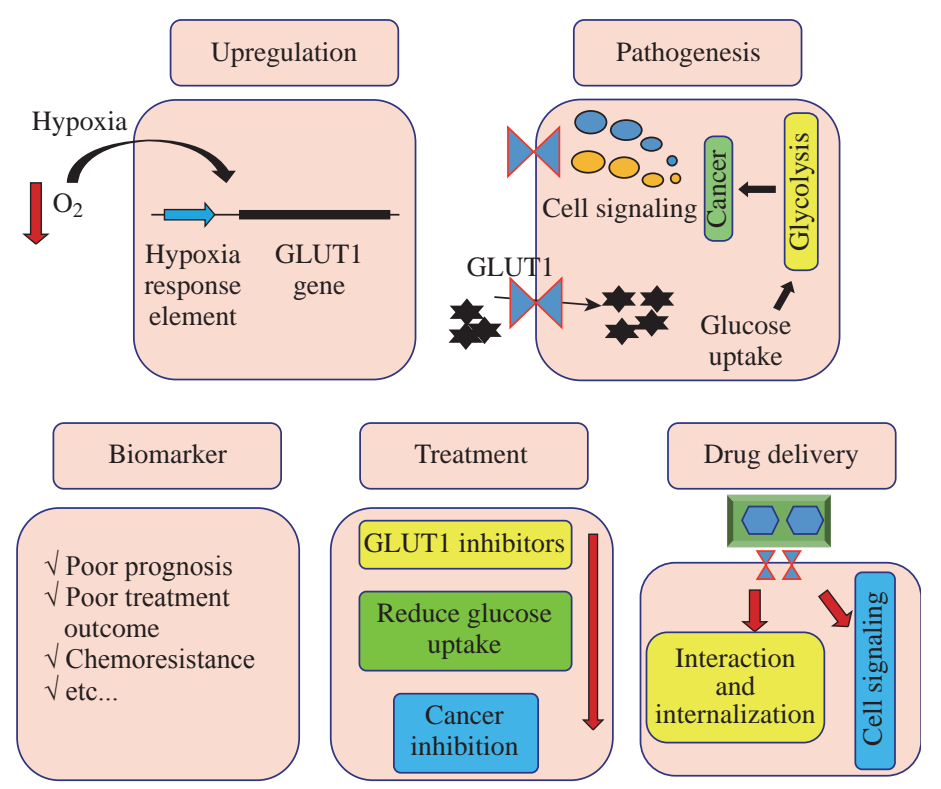

Fig. 1 GLUT1 gene is induced by hypoxia and promotes cancer progression. The overexpression of GLUT1 is associated with poor prognosis, and inhibition of this transporter reduces cancer growth. GLUT1 is considered as a target in drug delivery.

higher glucose uptake provides fuel for cancer growth that is consumed via anaerobic glycolysis [7]. Some evidence has shown that GLUT1 activates intracellular signaling pathways and induces cancer progression via the independent manner of glucose uptake [810]. However, the inhibition of this transporter reduces cancer growth and progression by reducing intracellular glucose. In addition to the pathologic role, GLUT1 overexpression is considered as the biomarker for cancer diagnosis, prognosis and classification. GLUT1 overexpression is associated with poor prognosis and treatment outcome [11, 12]. Recent efforts have tried to use GLUT1 as tools to deliver drugs in cancer treatment. The overexpression and cell surface presentation are two main characteristics of an antigen for targeting in drug delivery. The modification of nanocarrier with glucose leads to increase of the specific interaction between GLUT1 and modified-nanocarrier that promotes the efficacy of drug delivery. Several reports have used glucosemodified nanoparticles for drug delivery by targeting GLUT1 in the cancer and brain tissue [13-15]. In spite of the promising results, the authors did not explain the molecular mechanism of the action.

\section{Molecular Mechanisms}

As the first hypothetical mechanism, the central transport pores of GLUT1 which do not exceed 5 $\mathrm{nm}$ contribute to the internalization of the modified nanocarrier [16]. This mechanism puts forward the observation that the inhibition of GLUT1 transporter activity via inhibitors such as STF-31 and phloretin impaired the modified- nanoparticle internalization [17]. Because the size of nanocarrier is often over 10 $\mathrm{nm}$, the internalization of the modified nanocarrier via central transport pore is not explainable. As the second well-documented mechanism, nanocarrier-GULT1 complex is internalized via transporter-mediated endocytosis. Glucose-modified nanocarrier interacts with GULT1 and produces a complex that is uptaken via clathrin and caveolin mediated endocytosis [18]. A question is raised as to how the modified nanocarrier and GULT1 interaction activates the intercellular endocytosis systems including clathrin and caveolin. Therefore, the role of GULT1 is beyond a simple molecular gripper or brake for ceasing the nanocarrier movement. Herein, we hypothesize that GULT1 promotes nanocarrier internalization via mechanism other than a simple interaction.

\section{Controversial Aspects and New Hypothesis}

We propose that the interaction of nanoparticle and GLUT1 triggers intercellular processes that facilitates the internalization of nanoparticles such as cell signaling dependent endocytosis activation. This suggestion is based on the observations which showed GLUT1 regulated the MAPK and Src/FAK signaling 


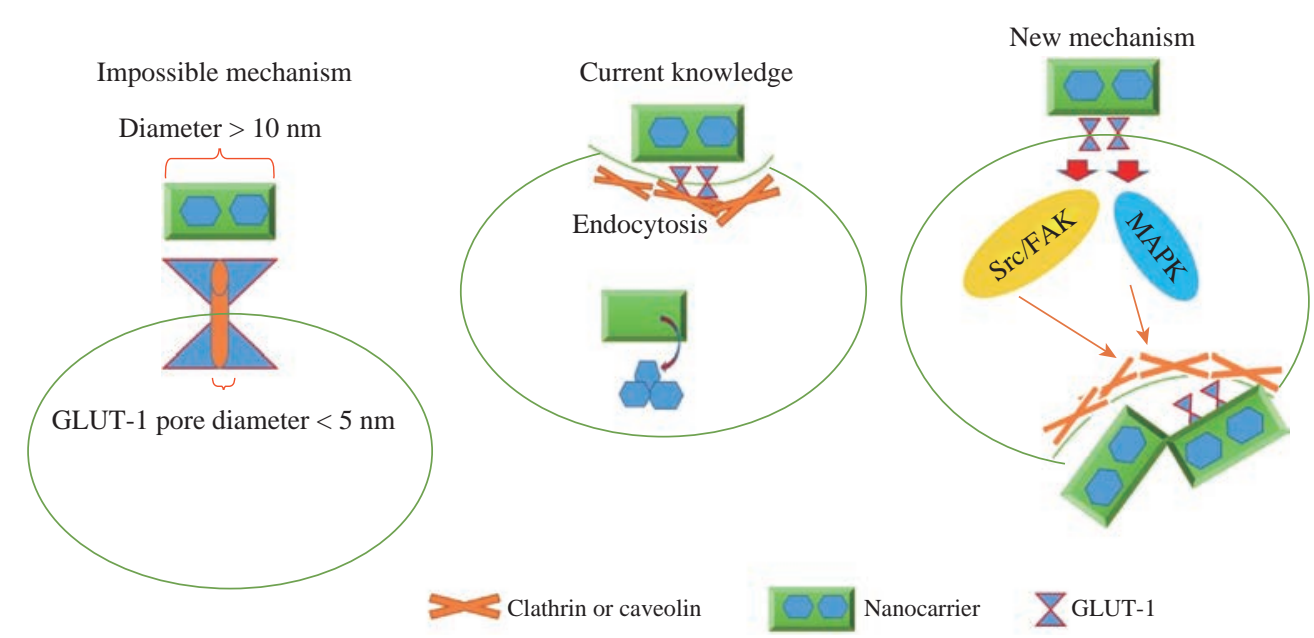

Fig. 2 The interaction of nanocarrier and GLUT1 may induce endocytosis system in cell signaling dependent manner.

pathways via glucose transport independent manner [19]. On the other hand, these signaling pathways modulate the clathrin-dependent and -independent endocytosis $[20,21]$. It is more possible that glucose conjugated nanocarrier and GLUT1 interaction induces the cell signaling pathway activation that triggers endocytosis in the cancer cell, resulting in drug internalization and delivery.

As the second controversial issue, if the translocation of nanocarrier is not mediated via GLUT1 pore, how the GLUT1 inhibitors impair nanoparticle internalization? Phloretin is a multifunctional drug with antioxidant and anti-inflammatory properties, and inhibits several intercellular enzymes and signaling pathways [22-25]. Hence, it remains unclear which properties of phloretin mediate the inhibition of nanoparticle internalization. We and other researchers have shown that NAC, an antioxidant agent could affect the nanoparticle internalization in the cell culture model [26, 27]. And at the same time, STF-31 interacts directly with GLUT1 and probably interferes with nanocarrier and GLUT1 interaction. Another study has shown that polyclonal anti-GLUT1 antibody has similar effects on glucose-coated superparamagnetic iron oxide nanoparticles [28]. Fig. 2 illustrates the current knowledge and novel aspects of new hypothesis.

\section{Epidermal Growth Factor Receptor as Another Witness}

Epidermal growth factor (EGF) receptor is another potential cell surface antigen to be targeted by nanocarrier. This receptor is overexpressed in breast cancer cells and has been targeted in drug delivery. EGF receptor activates several downstream signaling pathways. This receptor plays a pivotal role in drug cargo internalization as a component of the endocytosis system [29, 30]. We suggest that the GLUT1 role in drug delivery may occur in a similar way.

\section{Conclusions}

Taken together, we conclude that the modification of nanocarrier increases the GLUT1 dependent drug delivery. Modified nanocarrier- GLUT1 interaction increases nanocarrier internalization by two distinct ways: (1) Absorbance of nanocarrier to cell surface and thus ceasing its movements; and (2) activation of downstream signaling pathways and further endocytosis system activation.

\section{Perspective and the Future}

The evaluation of signaling pathways which are involved in the upstream of endocytosis system in the presence of modified-nanocarrier may be helpful to elucidate the mechanism of action. Moreover, the inhibition of these pathways by inhibitors may confirm the underlying mechanism.

Apart from that, the co-administration of nanocarrier and endocytosis inducers may be an effective method to increase drug delivery rate in cancer treatment.

\section{Conflicts of Interest}

The authors declare no conflict of interest. 


\section{References}

[1] S. Azzi, J.K. Hebda, and J. Gavard, Vascular permeability and drug delivery in cancers. Front Oncol, 2013, 3: 211.

[2] R. Bazak, Passive targeting of nanoparticles to cancer: A comprehensive review of the literature. Molecular and Clinical Oncology, 2014, 2(6): 904-908.

[3] T.D. Clemons, Distinction Between Active and Passive Targeting of Nanoparticles Dictate Their Overall Therapeutic Efficacy. Langmuir, 2018, 34(50): 1534315349.

[4] N. Muhamad, T. Plengsuriyakarn, and K. Na-Bangchang, Application of active targeting nanoparticle delivery system for chemotherapeutic drugs and traditional/ herbal medicines in cancer therapy: a systematic review. International Journal of Nanomedicine, 2018, 13: 3921.

[5] R. Bazak, Cancer active targeting by nanoparticles: a comprehensive review of literature. Journal of Cancer Research and Clinical Oncology, 2015, 141(5): 769-784.

[6] S.C. Pacheco-Velazquez, Energy Metabolism Drugs Block Triple Negative Breast Metastatic Cancer Cell Phenotype. Mol Pharm, 2018, 15(6): 2151-2164.

[7] K. Adekola, S.T. Rosen, and M. Shanmugam, Glucose transporters in cancer metabolism. Current Opinion in Oncology, 2012, 24(6): 650-654.

[8] C.D. Young, Activated Akt1 accelerates MMTV-c-ErbB2 mammary tumourigenesis in mice without activation of ErbB3. Breast Cancer Res, 2008, 10(4): R70.

[9] D. Avanzato, High USP6NL levels in breast cancer sustain chronic AKT phosphorylation and GLUT1 stability fueling aerobic glycolysis. Cancer Res, 2018, 78(13): 3432-3444

[10] R. Roy, AKT-dependent sugar addiction by benzyl isothiocyanate in breast cancer cells. Mol Carcinog, 2019.

[11] J.K. Blayney, Glucose transporter 1 expression as a marker of prognosis in oesophageal adenocarcinoma. Oncotarget, 2018, 9(26): 18518-18528.

[12] G.M.K. GabAllah, Validity and clinical impact of glucose transporter 1 expression in colorectal cancer. Saudi Journal of Gastroenterology: Official Journal of the Saudi Gastroenterology Association, 2017, 23(6): 348-356.

[13] R. Gromnicova, Glucose-coated gold nanoparticles transfer across human brain endothelium and enter astrocytes in vitro. PLoS One, 2013, 8(12): e81043.

[14] L. Zhang, Preparation and characterization of GLUT1mediated novel brain targeting magnetic nanoparticles. Letters in Drug Design \& Discovery, 2018, 15(12): 13081313.

[15] J. Li, Glucose-conjugated chitosan nanoparticles for targeted drug delivery and their specific interaction with tumor cells. Frontiers of Materials Science, 2014, 8(4): 363-372.

[16] A. Salas-Burgos, Predicting the three-dimensional structure of the human facilitative glucose transporter glut1 by a novel evolutionary homology strategy: Insights on the molecular mechanism of substrate migration, and binding sites for glucose and inhibitory molecules. Biophys J, 2004, 87(5): 2990-2999.

[17] L. Venturelli, Glucose is a key driver for GLUT1- mediated nanoparticles internalization in breast cancer cells. Sci Rep, 2016, 6: 21629.

[18] L. Kou, Transporter-guided delivery of nanoparticles to improve drug permeation across cellular barriers and drug exposure to selective cell types. Frontiers in Pharmacology, 2018, 9: 27.

[19] S. Oh, Glut1 promotes cell proliferation, migration and invasion by regulating epidermal growth factor receptor and integrin signaling in triple-negative breast cancer cells. BMB Rep, 2017, 50(3): 132-137.

[20] L.E. Johannessen, Epidermal growth factor receptor efficiently activates mitogen-activated protein kinase in HeLa cells and Hep2 cells conditionally defective in clathrin-dependent endocytosis. Exp Cell Res, 2000, 260(1): 136-145.

[21] X. Wu, FAK-mediated src phosphorylation of endophilin A2 inhibits endocytosis of MT1-MMP and promotes ECM degradation. Dev Cell, 2005, 9(2): 185-196.

[22] L. Han, Inhibitory effect of phloretin on alphaglucosidase: Kinetics, interaction mechanism and molecular docking. Int J Biol Macromol, 2017, 95: 520527.

[23] A. Takeno, Phloretin promotes adipogenesis via mitogenactivated protein kinase pathways in mouse marrow stromal ST2 cells. International Journal of Molecular Sciences, 2018, 19(6): 1772.

[24] K.R. Laderoute, 5'-AMP-activated protein kinase (AMPK) supports the growth of aggressive experimental human breast cancer tumors. The Journal of Biological Chemistry, 2014, 289(33): 22850-22864.

[25] B.M. Rezk, The antioxidant activity of phloretin: The disclosure of a new antioxidant pharmacophore in flavonoids. Biochem Biophys Res Commun, 2002, 295(1): 9-13.

[26] Z. Gholinejad, N-acetyl cysteine and metal nanoparticles internalization: A critical methodological aspect. Journal of Bionanoscience, 2018, 12(5): 700-704.

[27] J. Shi, Endothelial cell injury and dysfunction induced by silver nanoparticles through oxidative stress via IKK/ NF-kappaB pathways. Biomaterials, 2014, 35(24): 66576666.

[28] D. Barbaro, Glucose-coated superparamagnetic iron oxide nanoparticles prepared by metal vapour synthesis are electively internalized in a pancreatic adenocarcinoma cell line expressing GLUT1 transporter. PloS One, 2015, 10(4): e0123159-e0123159.

[29] A.M. Master, A. Sen Gupta, EGF receptor-targeted nanocarriers for enhanced cancer treatment. Nanomedicine, 2012, 7(12): 1895-1906.

[30] A. Tomas, C.E. Futter, and E.R. Eden, EGF receptor trafficking: Consequences for signaling and cancer. Trends in Cell Biology, 2014, 24(1): 26-34.

Copyright $\mathbb{C}$ Shiva Mehran, Zafar Gholinejad. This is an open-access article distributed under the terms of the Creative Commons Attribution License, which permits unrestricted use, distribution, and reproduction in any medium, provided the original author and source are credited. 